\title{
Objective and subjective outcomes following external dacryocystorhinostomy and inferior tear duct stenting in patients with acquired lacrimal drainage system obstruction
}

Julia Prinz

Universitätsklinikum Aachen

Niklas Plange

Universitätsklinikum Aachen

David Kuerten

Universitätsklinikum Aachen

Hannah Schellhase

Universitätsklinikum Aachen

Antonis Koutsonas

Universitätsklinikum Aachen

Peter Walter

Universitätsklinikum Aachen

Matthias Fuest ( $\square$ mfuest@ukaachen.de)

Universitätsklinikum Aachen

Keywords:

Posted Date: January 28th, 2021

DOI: https://doi.org/10.21203/rs.3.rs-153796/v1

License: (c) (i) This work is licensed under a Creative Commons Attribution 4.0 International License.

Read Full License 


\section{Abstract}

The authors have requested that this preprint be removed from Research Square. 